\title{
Implementing an Intervention to Improve Adverse Incident Reporting in the Hospital Setting: A Pilot Study
}

\author{
Keisuke Koeda \\ Iwate Ika Daigaku \\ Ryuji Uozumi \\ Kyoto University: Kyoto Daigaku \\ Fumiaki Takahashi \\ Iwate Ika Daigaku \\ Kuniaki Ogasawara \\ Iwate Ika Daigaku
}

Naomi Akiyama ( $\square$ nakiyama@iwate-med.ac.jp)

Iwate Ika Daigaku https://orcid.org/0000-0001-6365-9310

Research article

Keywords: patient safety, barrier to incident reporting, doctor, intervention, pilot study

Posted Date: November 9th, 2020

DOI: https://doi.org/10.21203/rs.3.rs-103166/v1

License: (c) (1) This work is licensed under a Creative Commons Attribution 4.0 International License. Read Full License 


\section{Abstract}

\section{Background}

To improve patient safety, it is needed to collect information related to adverse events and near-miss reports. This practice constitutes one of the strategies hospital managers use to understand the kinds of errors that occur at their hospitals.

This pilot study aimed to evaluate an intervention designed to improve error reporting rates among physicians.

Methods

The study was conducted at University Hospital A, where data were collected from April 2017 to March 2019. The intervention began in April 2018 and consisted of the four following steps: defining reporting standards, improving the incident reporting system, having the hospital administrators set clear goals and beginning a visualized feedback process, and achieving support and appropriate feedback as a leadership resource from the hospital administrator.

Results

Physicians' reporting rates were higher in FY 2018 than 2017. Particularly, differences began to occur in November of FY 2018 ( $p<0.05$, analyzed using Fisher's exact test). Further, the number of reports submitted by non-physicians increased by 900 in FY 2018 compared to those in FY 2017. Physicians tend to underreport minor incidents, but reports of near-miss events increased from 16 in FY 2017 to 106 in FY 2018. Reporting standards were focused on severe cases, but they had related treatment/procedure and drug, so that not only error cases but also near-miss cases were reported. Based on these results, the intervention effectively increased incident reporting rates among not only physicians, but also other staff members. In this regard, reporting barriers were broken when hospital administrators encouraged employees to submit incident reports.

\section{Conclusions}

Active intervention of hospital administrators, the executive class of the hospital, may encourage employees to submit incident reports, thus effectively removing reporting barriers.

\section{Background}

Fatal accidents resulting from medical errors range from $<5 \%$ to $>30 \%,{ }^{1}$ with estimates from developed nations suggesting that between $7.5 \%$ and $10.4 \%$ of patients in the acute care setting experience adverse drug events. ${ }^{2}$ A pilot study revealed $11.3 \%$ of hospitalized cases experienced adverse events in Japan. ${ }^{3}$ Using eight years of medical death-rate data from the United States, patient safety experts at Johns Hopkins calculated that more than 250,000 deaths per year were due to medical errors. ${ }^{1}$ The number of patient deaths caused by adverse events annually was estimated to be between 1,326 and 1,433 in Japan. ${ }^{4}$ To improve patient safety, it is needed to collect information related to adverse events and near-miss reports. This practice constitutes one of the strategies hospital managers use to understand the kinds of errors that occur at their hospitals.

Previous studies have focused on reporting barriers, and some studies have examined interventions for improving incident reporting rates. However, there are limited interventional studies. Most of the previous studies have focused on the factors hindering reporting behavior. Two previous studies suggested that organizational barriers (such as culture, the reporting system, and management behavior/system) and personal barriers (such as fear and accountability) are barriers to reporting behavior. ${ }^{5,6}$ Fear of consequences after error reporting was also a major perceived barrier. ${ }^{6,7}$ Other factors included the attitude of the administration department regarding legal issues, lack of effective medical error reporting systems, proper reporting forms, peer support for someone who has committed an error, and lack of knowledge regarding how to report and which error to report. ${ }^{6,8-10}$ In Evans et al.'s ${ }^{11}$ interventional study, various incidents were reported by the intervention units during the study period, with improved reporting rate among physicians from a low baseline. However, the study reported that there was considerable heterogeneity between reporting rates in different types of units. Regarding heterogeneity, lack of physician-initiated reports meant that feedback was limited to discussion of incidents generated in other areas. ${ }^{11}$ The most frequently stated barrier for physicians and nurses was lack of feedback. ${ }^{12}$ Safety culture includes seven aspects, and one of them is leadership. ${ }^{13}$ To reduce personal fear after reporting an incident, hospital administrators should be involved as a leadership resource. 
Thus, the current pilot study evaluated an intervention to increase incident reporting rates. The intervention was conducted in collaboration with hospital administrators with the aim of removing barriers to reporting incidents.

\section{Methods}

\section{Study setting}

This study was conducted at University Hospital A in northern Japan from fiscal year (FY) 2017 to FY 2018. The hospital contained approximately 1,160 beds and 2,650 staff members including 530 physicians (some of whom were dentists), 1,260 nurses, and 80 pharmacists. The hospital used an electronic reporting system that required all staff members to report all types of errors and near misses. As such, this study defined the scope of incident reports to include these. The reporting system operated through a local broadband network from within the hospital, with all reports reviewed by a medical safety management department (MSMD).

\section{Intervention methods}

Randomized control trials are likely to be challenging to conduct in this area, because finding sufficient units to randomize and suitable control groups will be challenging. ${ }^{14}$ This pilot study used a time-series design with comparisons before and after the intervention. The intervention examined in this pilot study consisted of four steps, the first of which was implemented in April 2018.

\section{Step 1: Define reporting standards beginning in April 2018}

The patient safety manual used at University Hospital A requires staff to report all incidents resulting from negligence, error, or failure. However, there was no standard requiring them to submit incident reports related to sentinel events. We, therefore, collaborated with MSMD members to define reporting standards for 33 topics and identified references related to adverse events, ${ }^{3}$ used Clavien-Dindo classification for complications, ${ }^{15}$ and investigated the reporting standards of several hospitals on their homepages. As shown in Table 1 , these were distributed across three categories Part 1 was related to surgery; Part 2 was related to medical events other than surgery; and Part 3 included all other events. These reporting standards were then described in the manual and announced during a patient safety meeting attended by approximately 120 risk managers, who were then required to work as departmental educators. Monthly meetings were held with these risk managers to report on their experiences in that regard.

\section{Step 2: Improvements to create a sufficient incident reporting system since September 2018}

University Hospital A was using its own electronic reporting system. However, clinical staff members asked the MSMD to improve this system because reporting forms were considered too long. A previous study suggested an appropriate reporting format in a single-page layout. ${ }^{11}$ The MSMD has an independent expert-trained department consisting of three nurses and one physician, and when there is lack of information on incident reports, they can call directly the incident reporter to obtain information. We therefore analyzed retrospective data and altered the question format from text input to select input, offered optional inputs rather than required inputs, and removed questions that were considered less important, reducing the total questions from 78 to 35 . These efforts were designed to alleviate the burdens on incident reporters. These improvements reduced the time usually needed to enter one report from 20 minutes to 5-10 minutes. MSMD announced improvements and ways to use this system in the risk managers' meeting, which was also useful for renotifying them about ways to access and use it.

\section{Step 3: Visualized feedback implemented by the hospital administrator since October 2018}

The MSMD prepared and published monthly documents concerning the submission status of incident reports from physicians inside the hospital, while the director referred to these documents when meeting with representatives of the clinical departments consisting of physicians. The published monthly documents include the number of incident reports from physicians from each department and a description of severe cases. Severe cases are included to discuss prevention policies as part of the hospital's risk management. 


\section{Step 4: Support and appropriate feedback as a leadership resource from the hospital administrator throughout FY 2018}

The director and deputy director related to MSMD stated strongly that incident reports are crucial for hospital administration and do not entail personal liability after reporting The deputy director (also one of the risk managers) explained the importance of incident reporting for preventing similar incidents during one of the risk-manager meetings. He specifically explained that staff members who reported incidents would not receive inappropriate feedback from their bosses consequently. This was also established as a hospital rule and listed in the patient safety manual.

\section{Data source and analysis}

The MSMD collected data from April 2017 to March 2018. In this context, the administrator of individual information deleted personal identifiers such as patient names, identification numbers, and the names of hospital staff members. Data were then given to this study's researchers. Data were varied, including reporting data (year/month/day), related personnel's occupations, impact on the patient outcomes due to the event, and type of reports (medication errors, transfusion errors, etc.). Differences in the reporting rates before and after the intervention each month were estimated with the use of Newcombe method ${ }^{16}$ and compared with the use of chi-square tests. Additionally, we compared the number and types of reports. All analyses were performed using SAS statistical software, version 9.4 and JMP version 12.0 (SAS Institute, Cary, NC).

\section{Ethics approval}

The MSMD collected incident reports for the hospital administration. This retrospective observational study analyzed this secondary data collected by the MSMD. The study was approved by the Ethics Committees at Iwate Medical University School of Medicine (Approval number: MH2018-073). The study involved only hospital staff members and not patients. No intervention was developed in this study. We obtained comprehensive agreements from participating hospital staff members and posted an official notice on the hospital's website from 30 September to 31 October 2018. Moreover, participating hospital staff members could refuse to the use of data during the recruitment period. Trials Registry identification number for this study is UMIN-CTR (UMIN000041087), and the data of registration 13 July 2020 (Retrospectively registered).

\section{Results}

\section{Characteristics of incident reports between FY 2017 and FY 2018}

Table 2 shows the characteristics of incident reports between FY 2017 and FY 2018. Incident reports of drugs were most frequently reported by staff, while those of treatment/procedure were most frequently reported by physicians. There were differences in reporter's occupation (staff, physicians) between FY 2017 and FY 2018. Among incident reports by staff, drug report rate of FY 2018 was higher than that of FY 2017.

Reports' impact on patients was also different between FY 2017 and FY 2018. Incident reports of FY 2018 were higher in near-miss both from staff and physicians.

\section{Intervention term and reporting numbers}

Table 3 shows the numbers and rates of incident reports from all staff members and physicians, with side-by-side comparisons for FY 2017 and FY 2018. Before conducting the intervention (FY 2017), physicians typically submitted around 30-40 reports per month, with the fewest in December and January (24 each; Table 3). The fewest overall number of physician-submitted reports (14) occurred in April of FY 2018, which was the same month the risk managers held their first meeting to reveal the new reporting standards for distribution to staff members. After this, reporting numbers substantially increased. Next, improvements to the incident reporting system were officially released in October, which is also when the hospital director began the visualized feedback process among physicians, whose incident reporting rates increased by $10 \%$ and higher since November of FY 2018. In particular, the highest monthly number of physician-submitted reports (79) was found in December, with the total reaching 641 in FY 2018. As shown in Table 3 and Figure 1, differences have occurred in all reporting rates since November of FY 2018. The differences in the physicians' reporting rates (FY 2018 minus FY 2017) were 6.2\%

Page $4 / 12$ 
(95\% confidence interval (Cl), 2.9 to 9.5; $\mathrm{P}<0.001)$ in November, 6.6\% (95\% Cl, 3.0 to 10.0; $\mathrm{P}<0.001)$ in December, 6.0\% (95\% Cl, 3.1 to 9.0; $\mathrm{P}<0.001)$ in January, $4.7 \%(95 \% \mathrm{Cl}, 1.0$ to $8.2 ; \mathrm{P}=0.012)$ in February, and $3.2 \%(95 \% \mathrm{Cl}, 0.1$ to $6.3 ; \mathrm{P}=0.045)$ in March.

\section{Discussion}

Our pilot intervention was associated with an increase in the number and rate of incident reports by physicians. Comparison from FY 2017 to FY 2018 revealed that the number of incident reports by both physicians and staff increased.

Physicians tended to report far fewer minor than major incidents, ${ }^{17,18}$ but near-miss reports were increasingly submitted, from 16 in FY 2017 to 106 in FY 2018. Reporting standards were focused on severe cases, but they had related treatment/procedure and drug, so that not only error cases but also near-miss cases were reported. Reasons for underreporting may include lack of knowledge, which includes how to report errors, what should be reported, and what kinds of errors to report. ${ }^{10,19}$ As such, reporting criteria were defined; staff members were informed about which incidents should be reported; and the MSMD could easily educate staff accordingly. Staff members were also pleased that incident reporting times were reduced. Announcement of reporting standards in our intervention brought knowledge and information about efficient reporting systems to physicians and all staff; this intervention led to the increase in the number of incident reports. However, physician submissions only increased to constitute $2.6 \%(95 \% \mathrm{Cl}, 1.7$ to 3.5$)$ more of the total in $\mathrm{FY}$ 2018 , thus reaching $9 \%$. The pilot intervention effectively increased the overall number of incident reports submitted by physicians but did not reach the aforementioned 10\% goal. Nevertheless, physicians submitted 241 more reports in FY 2018, with all employees submitting 871 A calculation of physician reporting rates revealed an increase in the number of all incident reports as denominators and the number of physicians reported as numerators. Hence, the overall physician reporting rates seemed small.

The pilot intervention also entailed a visualized condition feedback conducted on a monthly basis. However, effective safety feedback does not solely depend on publicized incident rates, but also requires timely, visible, and repeatable corrective actions and quality improvement processes. ${ }^{13}$ Concerning having hospital administrators provide support and feedback, previous studies have also shown that a patient safety manager can effectively reduce the fear of reporting. ${ }^{20}$ Most physicians believed that reduction in medical errors should be a national priority, but physicians believed that fear of medical malpractice is a barrier to reporting errors, and that greater legal safeguards are necessary for the success of mandatory reporting systems. ${ }^{19,21}$ Further, this study's intervention involved hospital managers, including the director and deputy director. Finally, it seemed that employees did not feel there was inappropriate feedback (including on financial and legal matters) when reporting incidents due to the direct involvement of the hospital administrator.

Its primary outcome was based on measuring the number of incident reports by physicians. Hence, it did not measure factors related to reporting barriers, including non-blaming, non-punitive, and non-fearful learning cultures. ${ }^{22,23}$ Despite these limitations, our results are meaningful. This study is the first to show that involving the executive department of a hospital is useful for implementing an intervention. Indeed, they should facilitate the hospital leadership by encouraging and rewarding employees when they report adverse and near-miss events.

\section{Conclusion}

This pilot study examined an intervention that helped remove incident reporting barriers to increase patient safety. The measures included establishment of (1) clear reporting standards, (2) an effective medical error reporting system, (3) feedback on what actions are/were taken, and (4) peer support/appropriate feedback as leadership endorsement from hospital administrators. A before-after intervention comparison revealed that all incident reporting rates increased (i.e., from physicians and all other staff members). Hospital administrators also encouraged employees to submit incident reports, thus effectively removing reporting barriers.

\section{Declarations}

\section{Ethics approval and consent to participate:}

The MSMD collected incident reports for the hospital administration. This retrospective observational study analysed this secondary data collected by the MSMD. The study was approved by Ethics Committees at Iwate Medical University School of Medicine (Approval number: MH2018-073). The study involved only hospital staff members, and not patients, and no intervention was developed in this study. We obtained comprehensive agreements from participating hospital staff members and posted an official notice on the hospital's website. Moreover, participating hospital staff members could refuse to the use of data during the recruitment period. The UMIN Clinical Trials 
Registry identification number for this study is UMIN-CTR (UMIN000041087), and the data of registration 13 July 2020 (Retrospectively registered)..

\section{Consent for publication:}

Not applicable.

\section{Availability of data and materials:}

The dataset generated and analysed during the current study are not publicly available. The dataset was permitted to be used within this study by Ethics Committees at Iwate Medical University School of Medicine because the dataset had the information from the field study and clinical staff. However, the dataset is available from the corresponding author on reasonable request.

\section{Competing interests}

The authors declare that they have no competing interests.

\section{Funding}

Grant-in-Aid for Young Scientists (B) (Number 20K18889) and Grant-in-Aid for Scientific Research (B) (Number 20H04147).

\section{Author's Contributions}

$\mathrm{KK}$ and $\mathrm{KO}$ conceived the study and supervised this work. NA was responsible for organising and coordinating the trial. NA, RU, and FT were responsible for data analysis and interpretation, drafting the manuscript tables and figure, and revisions to the manuscript. NA wrote the main body of the manuscript. All authors approved the final version of the manuscript. All authors contributed to writing the final manuscript.

\section{Acknowledgements}

We extend our appreciation to staff members of medical safety management department of Iwate Medical University Hospital. Naomi Akiyama received partial research support from ISPS KAKENHI Grant Numbers JP 20K18889. Ryuji Uozumi received partial research support from Grant-in-Aid for Scientific Research $20 \mathrm{H} 04147$.

\section{References}

1. Makary MA, Daniel M. Medical error-the third leading cause of death in the US. BMJ 2016;3:353.

2. World Health Organization. Summary of the evidence on patient safety: Implications for research. Research priority setting working group of the world alliance for patient safety [Internet]. 2008. Available at:

https://www.who.int/patientsafety/information_centre/20080523_Summary_of_the_evidence_on_patient_safety.pdf. Accessed May 28, 2020.

3. Ikeda S, Kobayashi M, Kaneko T, et al. Identification of adverse events in inpatients: results of a preliminary survey in Japan. Asian Pac J Dis Manag 2010;4(2):49-53.

4. Fujita S, lida S, Nagai Y, et al. Estimation of the number of patient deaths recognized by a medical practitioner as caused by adverse events in hospitals in Japan. Medicine 2017;96(39):1-5.

5. Vrbnjak D, Denieffe S, O'Gorman C, et al. Barriers to reporting medication errors and near misses among nurses: A systematic review. Int J Nurs Stud 2016;63:162-78.

6. Mirsadeghi A, Pazokian M. Barrier to reporting medication errors in Iran: A systematic review. Int J Med Rev 2015;2(4):317-21.

7. Yung HP, Yu S, Chu C, et al. Nurses' attitudes and perceived barrier to the reporting of medication administration errors. J Nurs Manag 2016;24:580-8. 
8. Soydemir D, Intepeler S, Mert H. Barriers to medical error reporting for physicians and nurses. West J Nurs Res 2016;39(10):1348-63.

9. Poorolajal J, Rezaie S, Aghighi N. Barriers to medical error reporting. Int J Prev Med 2015;6:97.

10. Kaldjian LC, Jones EW, Wu BJ, et al. Reporting medical errors to improve patient safety: a survey of physicians in teaching hospitals. Arch Intern Med 2008;168(1):40-6. doi:10.1001/archinternmed.2007.12.

11. Evans SM, Smith BJ, Esterman A, et al. Evaluation of an intervention aimed at improving voluntary incident reporting in hospitals. Qual Saf Health Care 2007;16:169-75.

12. Evans SM, Berry JG, Smith BJ, et al. Attitudes and barriers to incident reporting: a collaborative hospital study. Qual Saf Health Care 2006;15(1):39-43.

13. Sammer CE, Lykens K, Singh KP, et al. What is patient safety culture? A review of the literature. J Nurs Scholarsh 2010;42(2):156-165.

14. Parmelli E, Flodgren G, Fraser SG, et al. Interventions to increase clinical incident reporting in health care. Cochrane Database Syst Rev 2012;8(8):CD005609.

15. The Clavien-Dindo Classification [website]. https://www.assessurgery.com/clavien-dindo-classification/. Accessed 6 July 2020.

16. Hirose M, Regenbogen ES, Lipsitz S, et al. Lag time in an incident reporting system at a university hospital in Japan. Qual Saf Health Care 2007;16(2):101-4. doi:10.1136/qshc.2006.019851.

17. Smith KS, Harris KM, Potters L, et al. Physician attitudes and practices related to voluntary error and near-miss reporting. $J$ Oncol Pract 2014;10(5):350-7.

18. Rishoej RM, Hallas J, Juel Kjeldsen L, et al. Likelihood of reporting medication errors in hospitalized children: a survey of nurses and physicians. Ther Adv Drug Saf 2018;9(3):179-192.

19. Vrbnjak D, Denieffe S, O'Gorman C, et al. Barriers to reporting medication errors and near misses among nurses: a systematic review. Int J Nurs Stud 2016;63:162-78. doi:10.1016/j.jijnurstu.2016.08.019.

20. Robinson RA, Hohmann BK, Rifkin IJ, et al. Physician and public opinions on quality of health care and the problem of medical errors. Arch Intern Med 2002;162(19):2186-90.

21. Benn J, Koutantji M, Wallace L, et al. Feedback from incident reporting: information and action to improve patient safety. BMJ Qual Saf 2009;18(1):11-21.

22. Nieva VF, Sorra J. Safety culture assessment: a tool for improving patient safety in healthcare organizations. Qual Saf Health Care 2003;12(2):17-23.

\section{Tables}

Table 1. Defining reporting standards 
Part 1: Related to surgery

1. Misidentification of patient, treatment site, method, or usage

2. Items remaining in the body after surgery, such as gauze or needles

3. Unexpected over-bleeding not associated with surgery

4. Infarction, rupture, perforation, or rupture associated with invasive procedure

5. Serious complications associated with surgery

6. Nerve palsy that develops after surgery

7. Blood clotting after surgery

8. Cardiopulmonary arrest or death during surgery

9. Anesthesia accident involving intubation and/or extubation

10. Side-effect associated with anesthesia

11. Damage associated with malfunction or corruption

12. Emergency surgery

13. Re-surgery associated with complications

14. Re-surgery during admission or within seven days after discharge

15. Death within 30 days of surgery

Part 2: Other than surgery (treatment, exam, etc.)

1. Death, cardiopulmonary arrest, or respiratory arrest without prediction

2. Unexpected over-bleeding

3. Sudden change in vitals associated with contrast medium, medication, transfusion, or blood product 
4. Adverse effects or possible adverse effects to the patient associated with

misdiagnosis, negligence, or delayed treatment

5. Severe damage associated with overlooking medical test results

6. Unexpected prolonged hypo-oxygen in a full-term newborn infant

7. Brain damage or fractures associated with falling in hospital

8. Sudden changes in vitals or respiratory arrest associated with aspiration or providing prohibited meals

9. Severe burn associated with treatment

10. Severe side-effects or complications associated with treatment, examinations, or rehabilitation

11. Severe damage from pressure ulcer or vascular leakage

\section{Part 3: Others}

1. Suicide or suicide attempt by an inpatient

2. Severe complications experienced by an inpatient (brain infraction, heart attack, pulmonary thrombus, or cerebrovascular disease)

3. Violence from a patient

4. Disappearance of an inpatient

5. Stolen or lost patient baggage

6. Bringing dangerous goods to the hospital or experiencing suffocation due to foreign matter inside the hospital

7. Accidental burning or explosion 


\begin{tabular}{|c|c|c|c|c|c|c|}
\hline & \multicolumn{3}{|l|}{ By staff } & \multicolumn{3}{|c|}{ By physicians } \\
\hline & FY2017 & FY2018 & P_value & FY2017 & FY2018 & P_value \\
\hline & $n=5,875$ & $\mathrm{n}=6,505$ & & $n=400$ & $n=641$ & \\
\hline \multicolumn{7}{|l|}{ Type incident report ${ }^{a}$} \\
\hline Drug & $1,580(26.9)$ & $1,788(27.5)$ & $<0.001$ & $35(8.8)$ & $80(12.5)$ & 0.001 \\
\hline Drainage tube & $1,227(20.9)$ & $1,165(17.9)$ & & $22(5.5)$ & $24(3.7)$ & \\
\hline Treatment/procedure & $694(11.8)$ & 707 (10.9) & & $236(59.0)$ & $303(47.3)$ & \\
\hline Nursing care & $793(13.5)$ & $899(13.8)$ & & $10(2.5)$ & $10(1.6)$ & \\
\hline Examination & $499(8.5)$ & $524(8.1)$ & & $28(7.0)$ & $53(8.3)$ & \\
\hline Medical devices and other equipment & $278(4.7)$ & $298(4.6)$ & & $23(5.8)$ & $41(6.4)$ & \\
\hline Blood transfusion & $44(0.7)$ & $66(1.0)$ & & $3(0.8)$ & $8(1.2)$ & \\
\hline Others & $760(12.9)$ & $1,058(16.3)$ & & $43(10.8)$ & $122(19.0)$ & \\
\hline \multicolumn{7}{|l|}{ Impact of report on patient ${ }^{b}$} \\
\hline Near miss & $728(12.4)$ & $1,130(17.4)$ & $<0.001$ & $16(4.0)$ & $106(16.5)$ & $<0.001$ \\
\hline Error & $5,147(87.6)$ & $5,375(82.6)$ & & $384(96.0)$ & 535 (83.5) & \\
\hline
\end{tabular}

${ }^{a}$ using $\chi^{2}$ test; ${ }^{b}$ Fisher's exact test

Table 3. The number and rate of incident reports by physicians

\begin{tabular}{|c|c|c|c|c|c|c|c|c|c|c|c|c|c|c|c|c|c|c|c|}
\hline & \multicolumn{9}{|c|}{ FY2017 } & \multicolumn{9}{|c|}{ FY2018 } & \multirow[t]{3}{*}{ p_value } \\
\hline & \multirow{2}{*}{$\begin{array}{l}\text { All } \\
\mathrm{N}\end{array}$} & \multicolumn{4}{|c|}{ from staffs } & \multicolumn{4}{|c|}{ from physicians } & \multirow{2}{*}{$\begin{array}{l}\text { All } \\
\mathrm{n}\end{array}$} & \multicolumn{3}{|c|}{ from staffs } & \multicolumn{5}{|c|}{ from physicians } & \\
\hline & & $n$ & ( & $\%$ & ) & $\mathrm{n}$ & ( & $\%$ & ) & & $\mathrm{n}$ & ( & $\%$ & ) & $\mathrm{n}$ & ( & $\%$ & ) & \\
\hline April & 524 & 487 & ( & 92.9 & ) & 37 & ( & 7.1 & ) & 346 & 332 & ( & 96.0 & ) & 14 & ( & 4.0 & ) & 0.076 \\
\hline May & 532 & 494 & ( & 92.9 & ) & 38 & ( & 7.1 & ) & 507 & 477 & ( & 94.1 & ) & 30 & ( & 5.9 & ) & 0.453 \\
\hline Jun & 588 & 552 & ( & 93.9 & ) & 36 & ( & 6.1 & ) & 596 & 545 & ( & 91.4 & ) & 51 & ( & 8.6 & ) & 0.119 \\
\hline Jul & 534 & 492 & ( & 92.1 & ) & 42 & ( & 7.9 & ) & 599 & 548 & ( & 91.5 & ) & 51 & ( & 8.5 & ) & 0.745 \\
\hline August & 503 & 469 & ( & 93.2 & ) & 34 & ( & 6.8 & ) & 616 & 580 & ( & 94.2 & ) & 36 & ( & 5.8 & ) & 0.538 \\
\hline September & 497 & 467 & ( & 94.0 & ) & 30 & ( & 6.0 & ) & 599 & 552 & ( & 92.2 & ) & 47 & ( & 7.8 & ) & 0.286 \\
\hline October & 600 & 563 & ( & 93.8 & ) & 37 & ( & 6.2 & ) & 744 & 684 & ( & 91.9 & ) & 60 & ( & 8.1 & ) & 0.204 \\
\hline November & 528 & 498 & ( & 94.3 & ) & 30 & ( & 5.7 & ) & 604 & 532 & ( & 88.1 & ) & 72 & ( & 11.9 & ) & $<0.001$ \\
\hline December & 410 & 386 & ( & 94.1 & ) & 24 & ( & 5.9 & ) & 634 & 555 & ( & 87.5 & ) & 79 & ( & 12.5 & ) & $<0.001$ \\
\hline January & 582 & 558 & ( & 95.9 & ) & 24 & ( & 4.1 & ) & 612 & 550 & ( & 89.9 & ) & 62 & ( & 10.1 & ) & $<0.001$ \\
\hline February & 483 & 447 & ( & 92.5 & ) & 36 & ( & 7.5 & ) & 569 & 500 & ( & 87.9 & ) & 69 & ( & 12.1 & ) & 0.013 \\
\hline March & 494 & 462 & ( & 93.5 & ) & 32 & ( & 6.5 & ) & 720 & 650 & ( & 90.3 & ) & 70 & ( & 9.7 & ) & 0.046 \\
\hline Sum & 6275 & 5875 & ( & 93.6 & ) & 400 & ( & 6.4 & ) & 7146 & 6505 & ( & 91.0 & ) & 641 & ( & 9.0 & ) & $<0.001$ \\
\hline
\end{tabular}


14.0

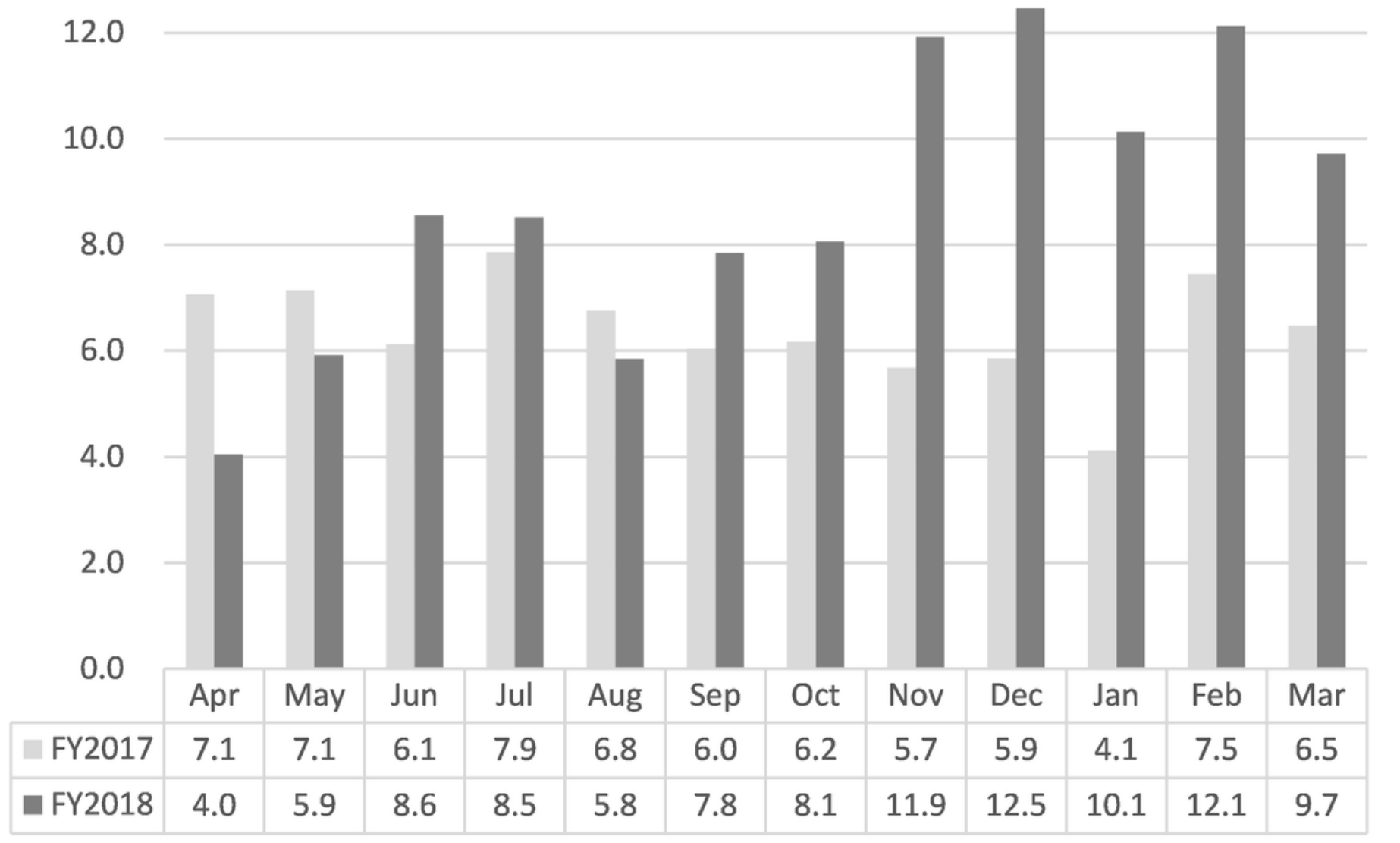

Figure 1

Incident reporting rates among physicians 
14.0

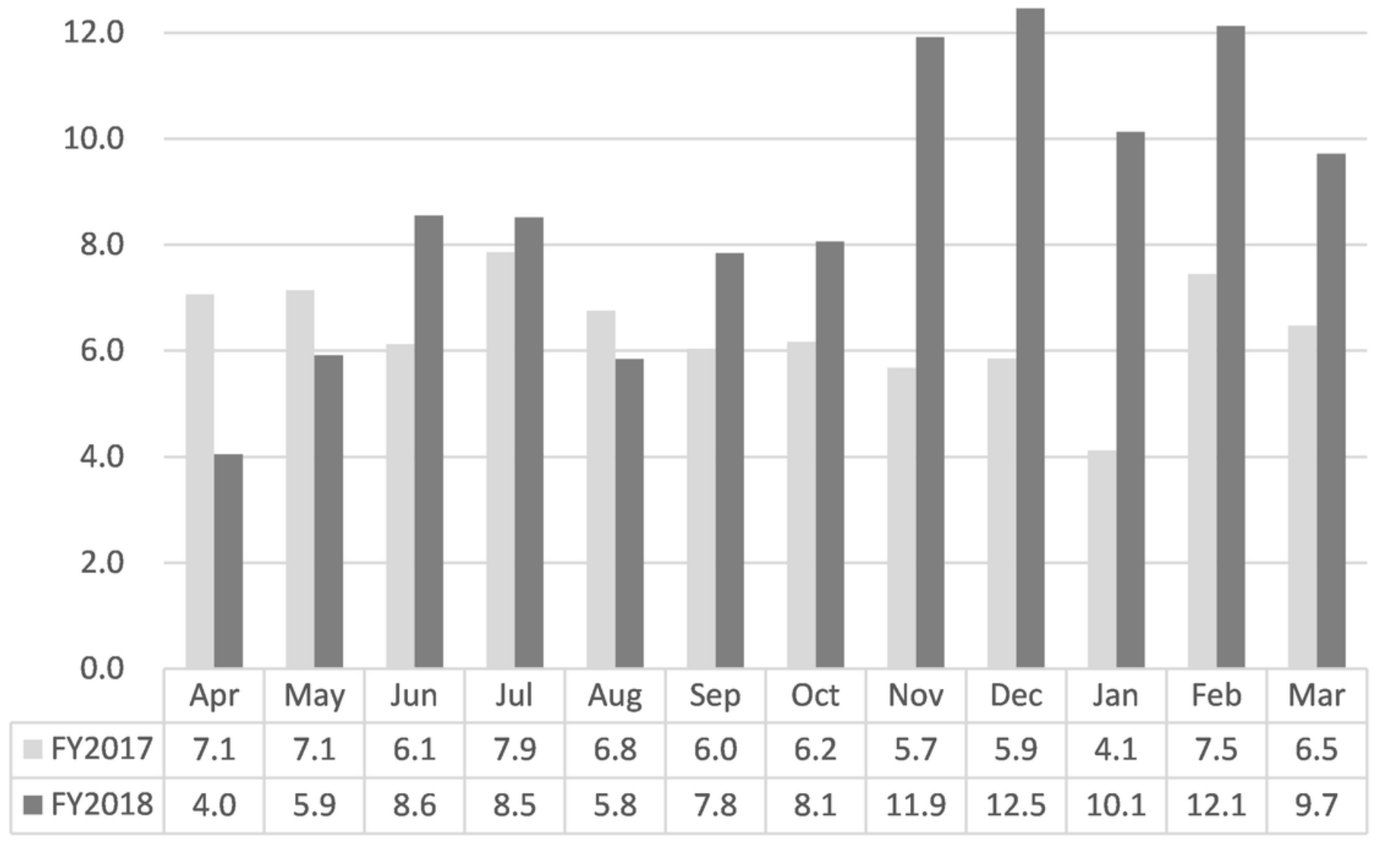

Figure 1

Incident reporting rates among physicians 